\title{
Clivus Chondroid Chordoma
}

National Cancer Institute

\section{Source}

National Cancer Institute. Clivus Chondroid Chordoma. NCI Thesaurus. Code C5426.

A slow-growing malignant bone tumor arising from the remnants of the notochord and occurring in the clivus. It is characterized by a lobulated growth pattern, myxoid stroma formation, the presence of physaliphorous cells and cartilage. 